\title{
The evolution of the treatment of traumatic cerebrovascular injury during wartime
}

\author{
A review \\ Randy S. Bell, M.D. ${ }^{1,3}$ Robert D. Ecker, M.D. ${ }^{1,3}$ Meryl A. Severson III, M.D., ${ }^{1}$ \\ John E. Wanebo, M.D., ${ }^{1}$ Benjamin Crandall, D.O., ${ }^{2}$ and Rocco A. Armonda, M.D. ${ }^{1,3,4}$ \\ Departments of ${ }^{1}$ Neurosurgery and ${ }^{2}$ Radiology, National Naval Medical Center; ${ }^{3}$ Department of \\ Neurosurgery, Uniformed Services University of the Health Sciences, Bethesda, Maryland; and ${ }^{4}$ Department \\ of Neurosurgery, Walter Reed Army Medical Center, Washington, DC
}

\begin{abstract}
The approach to traumatic craniocervical vascular injury has evolved significantly in recent years. Conflicts prior to Operations Iraqi and Enduring Freedom were characterized by minimal intervention in the setting of severe penetrating head injury, in large part due to limited far-forward resource availability. Consequently, sequelae of penetrating head injury like traumatic aneurysm formation remained poorly characterized with a paucity of pathophysiological descriptions. The current conflicts have seen dramatic improvements with respect to the management of severe penetrating and closed head injuries. As a result of the rapid field resuscitation and early cranial decompression, patients are surviving longer, which has led to diagnosis and treatment of entities that had previously gone undiagnosed. Therefore, in this paper the authors' purpose is to review their experience with severe traumatic brain injury complicated by injury to the craniocervical vasculature. Historical approaches will be reviewed, and the importance of modern endovascular techniques will be emphasized. (DOI: 10.3171/2010.2.FOCUS1025)
\end{abstract}

$\begin{array}{lccc}\text { KEY WoRDS } & \text { traumatic vasospasm } & \text { endovascular } & \bullet \\ \text { traumatic aneurysm } & \bullet & \text { penetrating head trauma }\end{array}$

$\mathrm{T}$ HE effects of modern warfare and its constituent military-grade weaponry have resulted in considerable craniocervical injuries. Nowhere is this more poignant than the injuries suffered by US servicemen and women during OIF and OEF over the last 6 years. Although the loss of life and limb is tragic, the medical advancements achieved during efforts to save individuals are substantial and apply to every field of medicine. One particular example includes the advances in approaches to traumatic craniocervical vascular injury made by military neurosurgical personnel. Improvements in endovascular technologies (coils, microcatheters, and liquid embolic agents) have shifted what was traditionally a treatment paradigm dominated by open surgical techniques to one in which a multidisciplinary approach is the standard of care. The purpose of this paper is to review the historic approach to these lesions and subsequently to summarize the most recent experience with the largest population of patients with traumatic craniocervical vascular injury

\footnotetext{
Abbreviations used in this paper: $\mathrm{DS}=$ digital subtraction; $\mathrm{OEF}=$ Operation Enduring Freedom; OIF = Operation Iraqi Freedom; SAH $=$ subarachnoid hemorrhage; $\mathrm{TCD}=$ transcranial Doppler.
}

ever reported. For the purposes of discussion, this paper will focus on 2 subtypes of cerebrovascular injury: traumatic aneurysms and traumatic vasospasm.

\section{Historical Approach to Neurovascular Trauma}

\section{Traumatic Aneurysms}

The true incidence of traumatic aneurysms due to the lethal nature of a penetrating missile or fragment is largely speculative. Estimates in the setting of penetrating head injury range from 5 to $40 \% .^{1,2,4,10,16-18}$ The lower end of this range is likely an underestimate if one considers that 1) many patients, both civilian and military, do not survive to reach hospital care; and 2) standard of care at many institutions does not include mandatory cerebral angiography for all penetrating head injuries. Overall, traumatic aneurysms comprise $1 \%$ of all forms of intracranial aneurysms.

Prior to and long after the advent of cerebral angiography by Egas Moniz in 1927, the diagnosis of traumatic aneurysms was observational, relying heavily on what was seen during surgical exploration. The advent 


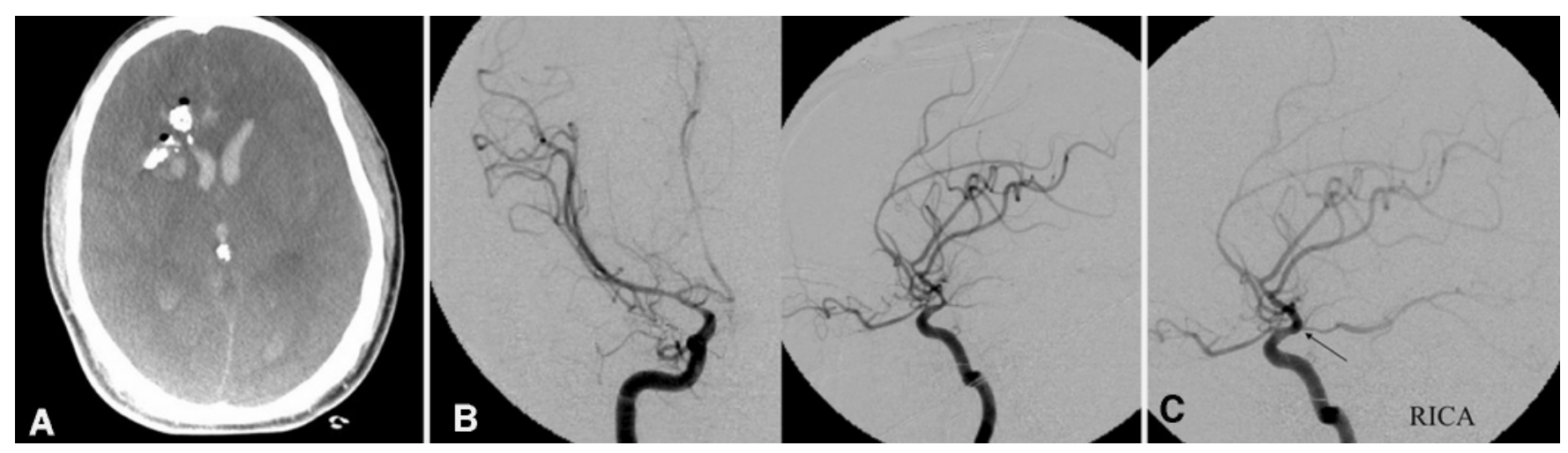

FIG. 1. A: Penetrating head injury accompanied by intraventricular hemorrhage. B: Anteroposterior (left) and lateral (right) angiograms of the right internal carotid artery (ICA) demonstrating severe supraclinoid and proximal middle cerebral artery vasospasm. C: Lateral angiogram of the right ICA (RICA) following balloon angioplasty showing resolution of the vasospasm.

of DS technology made noninvasive diagnosis possible and accurate. ${ }^{3,8,13,21,24,28}$ Although several small studies within the civilian sector exist concerning these entities, it has been through large-scale military conflict that advances in diagnosis, natural history, and treatment have occurred. . $2,4,10,17^{2}$

Groundbreaking work in the area of traumatic aneurysms was first reported in 1988 following the Iran-Iraq War. Aarabi ${ }^{2}$ analyzed his experience with 255 head-injured patients who received diagnostic angiography. What is important to remember about this remarkable population is that these were the individuals who survived their injury, often without the protective benefits of a helmet, and survived their subsequent transport (often protracted through hostile territory) through rough Iranian terrain. This work was the first to characterize the wounding patterns that, if present, correlate strongly with the concomitant presence of cerebrovascular injury. Specifically, fragment or missile penetrations through orbitofrontal and/or pterional windows were associated with the formation of traumatic aneurysms. Although only 7 individuals were diagnosed with injury in this group, all were diagnosed more than 10 days after their injury, and all were treated with open surgical clip exclusion.

A subsequent study from the same conflict on a larger population of head-injured patients further characterized this disease entity. Amirjamshidi et al. ${ }^{4}$ analyzed their experience with more than 1100 head injuries, including outcomes associated with surgical intervention. They identified within this population a uniform subpopulation of individuals who received diagnostic angiograms within a consistent time frame. Within this group, 5.7\% of those studied had traumatic aneurysms and were subsequently treated by either open surgical clipping or observation.

\section{Traumatic Vasospasm}

Although the natural history of aneurysmal SAHinduced vasospasm has been well characterized, several questions remain concerning the same for posttraumatic vasospasm. What is known is that the incidence of vasospasm after trauma increases with the amount of traumatic SAH present on the admission head $\mathrm{CT},{ }^{31,32,36}$ that the incidence of vasospasm is inversely proportional to admission Glasgow Coma Scale scores, ${ }^{6,36,37}$ that the vasospasm can be reliably monitored with TCD ultrasonography, $7,31,32,34$ and the time course of spasm in this setting is variable. ${ }^{6,31,32,36,37}$ There is, however, continued debate concerning whether, like aneurysmal SAH vasospasm, traumatic vasospasm causes delayed ischemic neurological deficits and worsens outcomes. ${ }^{6,15,32}$ It is because of this debate that, prior to 2006, very few studies were available that evaluated the effect of endovascular treatment interventions in this setting. ${ }^{12,34}$ Overall and with respect to military trauma populations, there are no studies prior to OIF and OEF evaluating the incidence or importance of traumatic vasospasm in a wartime trauma population.

\section{Operation Iraqi Freedom}

Operation Iraqi Freedom began on April 18, 2003. Since that time, the vast majority of in-theater casualties, following their initial resuscitation and surgical stabilization, were treated at the Walter Reed Army Medical Center and the National Naval Medical Center, Bethesda. A recent study chronicled the extent of severe CNS injury that survived to reach the continental US. ${ }^{11}$ Between April 2003 and April 2008, 513 patients transferred from OIF/OEF to National Naval Medical Center and Walter Reed Army Medical Center required neurosurgical evaluation. In this group, 408 patients presented with severe head trauma, with the majority suffering a penetrating head injury from blast. Several salient observations were made from the study of general head injury. First, early decompressive craniectomy (within the first 2-4 hours from injury) was successful in mitigating the effects of swelling and increased intracranial pressure during the long air evacuation transfer to the continental US. Second, the relative incidences of pulmonary embolism, cerebrovascular injury, and associated spinal column injury exceeded previously published civilian reports in head injury populations. Third, outcomes at all time points were directly proportional to the extent of initial neurological and systemic injury.

Of the many observations made, the most striking was the incidence of traumatic cerebrovascular injury. More than one-third of those presenting with severe head 


\section{Evolution of treatment for traumatic cerebrovascular injury}

trauma suffered concomitant vascular injury. This represents a significant increase from the 5-20\% reported previously in similar studies. ${ }^{1,2,4,18}$ Reasons for this observed increase include improved far-forward care (immediate resuscitation and early cranial decompression) resulting in longer survival as well as improved diagnostic techniques (DS angiography with 3D reconstruction). The following discussion will address the most current approaches to and treatment of traumatic vasospasm and traumatic aneurysms.

\section{Traumatic Vasospasm}

Vasospasm can be simply defined as a reduction in the caliber of a blood vessel, usually in response to an external stimulus that results in increased flow velocities (Fig. 1). Although clinically silent in many circumstances, vasospasm can result in delayed ischemic neurological deficits. ${ }^{32}$

Prior to OIF, the indication to perform diagnostic cerebral angiography in penetrating head injury populations was limited to those with penetrating head injury in whom traumatic aneurysms were suspected. Because a considerable proportion of those studied (in our population) had either concomitant or isolated vasospasm, we expanded our indications to include all those with penetrating head injuries, all those who suffered either a closed or penetrating head injury as a result of blast, and all those in whom increased cerebral blood flow velocities were documented by TCD ultrasonography.

Initial results in this population were reported in $2006 .{ }^{6}$ Specifically, the incidence of vasospasm in our population approached $50 \%$ and was associated with the presence of traumatic aneurysms and intracranial blood of any type. The incidence of vasospasm peaked at 14 days and was reliably followed by TCD ultrasonography. Roughly two-thirds of those with vasospasm received either balloon angioplasty or direct intraarterial infusions of nicardipine to mitigate the vasospasm. While those aggressively treated did not show statistical improvement with respect to early Glasgow Outcome Scale scores when compared with those treated conservatively, there was a statistical reduction in the vasospasm as measured by TCD ultrasonography. Overall, those with vasospasm had statistically lower early and late Glasgow Outcome Scale scores than those who did not have vasospasm.

While previous studies characterized the formation of vasospasm following trauma, ${ }^{19,20,22,23,29,31,32,36,37}$ few were able to correlate delayed ischemic deficits and outcomes with its presence, ${ }^{15,31,32,37}$ and even fewer described the safety or efficacy of endovascular interventions in this setting. ${ }^{12}$ In addition, while not specifically correlated with vasospasm occurrence, there was a considerable predominance of blast-induced head injury in our population. In approximately $10 \%$ of those with vasospasm, no penetrating injury was present. We theorized that the blast overpressure wave that accompanies all explosions may be responsible, at least in part, for the duration and severity of the vasospasm seen in our population. Experiments conducted in porcine systems (unpublished data) confirm that isolated blast overpressure can cause early
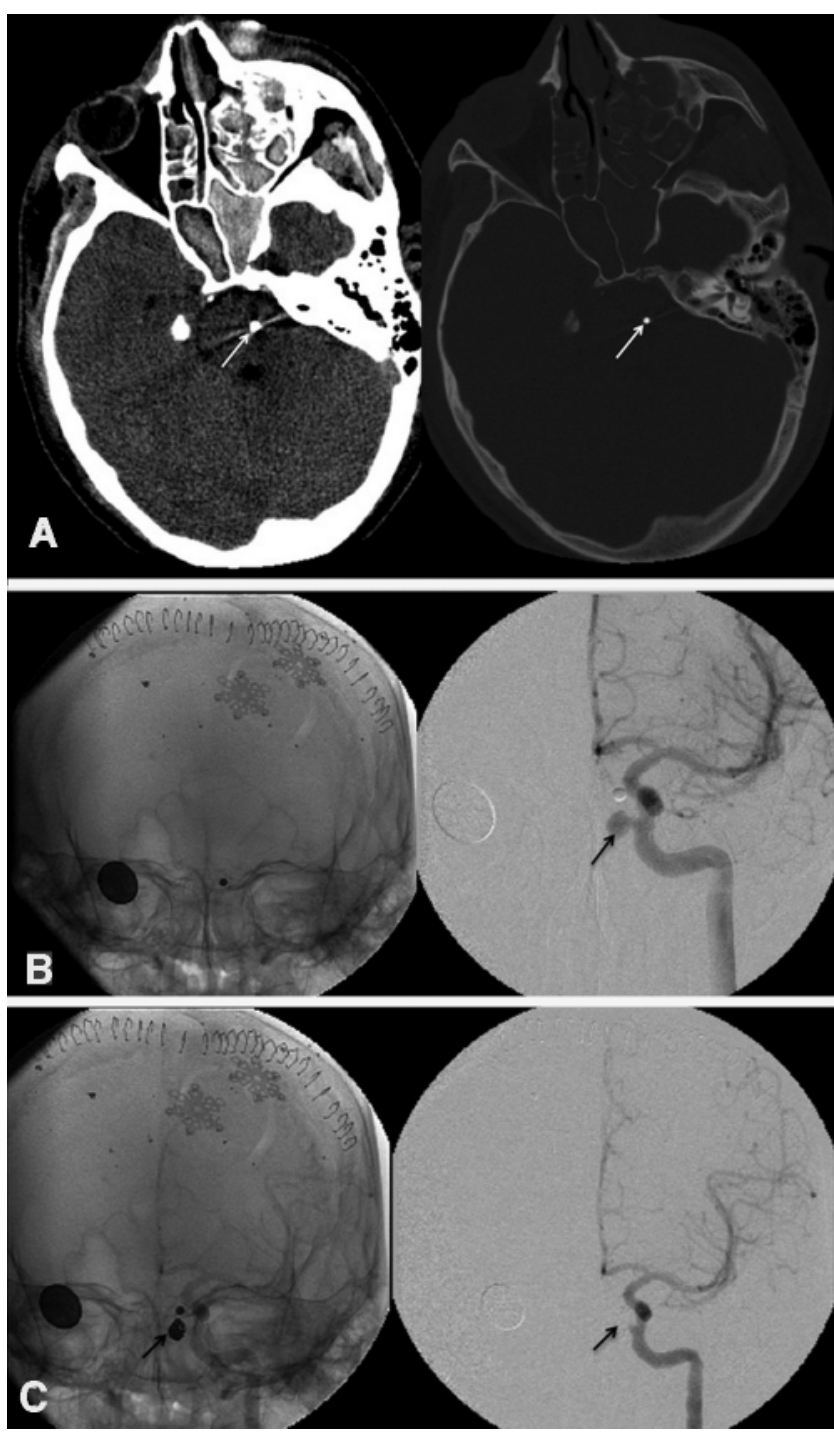

FIG. 2. A: Head CT scans with soft-tissue (left) and bone (right) windows revealing a penetrating facial injury by a small spherical fragment that traversed the sphenoid sinus and came to rest within the brainstem (arrows). This patient presented to the continental US with severe epistaxis that resulted in a code arrest. B: Native (left) and anteroposterior (AP) DS (right) angiograms of the left ICA demonstrating a traumatic aneurysm projecting medially from the proximal cavernous segment of the ICA (arrow). C: Native AP (left) and AP DS (right) angiograms of the left ICA following successful coil occlusion of the traumatic aneurysm (arrows). The patient made a full recovery and returned to active duty.

vasospasm (within 12 hours), although long-term studies have not been completed..$^{9}$ Overall, it is possible that the vasospasm seen in this population differs mechanistically from that seen in general civilian and military nonblast trauma.

\section{Traumatic Aneurysms}

Traumatic aneurysms result from injury to the arterial wall from an external source (Figs. 2 and 3). Connective tissue remnants or frank clot form the components of the "dome" of the aneurysm. It is the unpredictable 

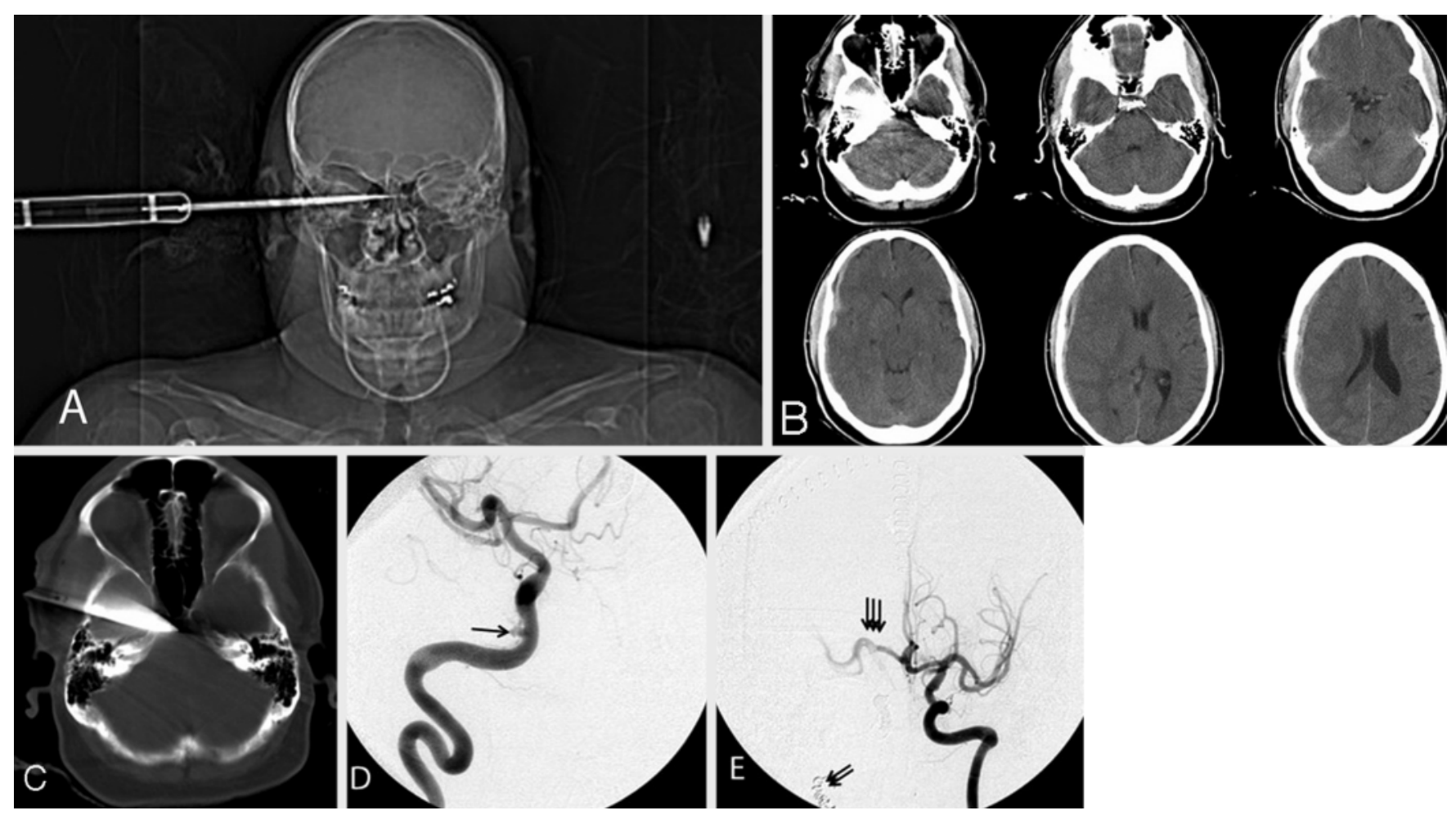

FIG. 3. A: Scout image obtained from a CT scan in a patient, revealing a penetrating knife wound to the head B: Axial CT images of the brain revealing subdural hematoma and evidence of SAH. C: Axial CT scan of the brain, bone window, revealing the course of the knife through the middle cranial fossa with the tip lodging medially near the inferior aspect of the cavernous sinus. D: Oblique DS angiogram of the right ICA revealing a traumatic aneurysm at the proximal portion of the cavernous segment of the ICA (arrow). E: Digital subtraction angiogram of the left ICA showing right ICA occlusion (double arrows) with excellent cross-filling of the right anterior cerebral artery and middle cerebral artery via a patent anterior communicating artery (triple black arrows). The patient made an excellent recovery.

rupture rate of this type of aneurysm that makes early treatment important. That said, unlike spontaneous saccular aneurysms for which a significant body of published information is available concerning their natural history and treatment,,$^{14,25-27,35}$ very little of the same data were available for traumatic aneurysms prior to 2010 . Recommendations based on available case series $(<80$ cases $)$ were made by the Head Trauma Society in the Guidelines for the Management of Vascular Complications of Penetrating Head Injury. ${ }^{5}$ At no more than the level of Option, the Society recommends diagnostic cerebral angiography in most cases, and recommends treatment, although the timing and type of treatment remain vague. Prior to the current conflict, open surgical approaches mainly consisting of clip exclusion of the diseased arterial segment were the mainstay of treatment, with little to no experience in endovascular options.

Operations Iraqi and Enduring Freedom have seen the largest concentration of penetrating and closed head injury in US servicemen and women since the Vietnam War. As previously stated, as a result of improved imaging technology and heightened awareness, vascular injuries were identified in nearly one-third of those injured, with traumatic aneurysms seen in nearly one-third of those studied with DS angiography. The results of our analysis of this population were published in January $2010 .^{12}$ Overall, 64 arterial injuries were seen in 187 pa- tients studied with DS angiography. Fifty traumatic aneurysms (31 traumatic intracranial aneurysms and 19 traumatic extracalvarial aneurysms) were seen. While some aneurysms were treated with clip exclusion at the time of the original cranial intervention, many presented in a delayed fashion at the time of the first angiographic study (on average 10 days after injury).

Several conclusions were reached as a result of this study. First, the pattern of rupture of these aneurysms was not consistent, but trended toward larger aneurysm size. Second, some traumatic aneurysms (on average, 2 $\mathrm{mm}$ in size) healed without treatment. Lastly, endovascular options (coil occlusion, liquid embolic agents, and stent-buttressed coil embolization) are viable and safe. In most cases they can prevent or delay an additional open surgical exploration in the acute setting where sequelae of head injury (high intracranial pressure and scarring) can hamper surgery and worsen outcome.

\section{Lessons Learned and Recommendations for Future Conflicts}

The impact of head trauma accompanied by neurovascular injury has been significant. Advances in helmet and body armor technology, far-forward systemic resuscitation, early cranial decompression, and a high degree 


\section{Evolution of treatment for traumatic cerebrovascular injury}

of suspicion with an algorithmic approach to performing DS angiography have improved outcomes in this highrisk population.

With respect to future conflicts, it is imperative that an infrastructure be maintained to aggressively manage these conditions. Neurosurgeons and neurocritical care specialists must maintain a high degree of suspicion for these injuries. This approach should include mandatory DS angiography performed in all patients with penetrating brain injury, and most patients with traumatic brain injury from blast. As technology improves, moving higher resolution portable angiographic suites far-forward (Level III) may improve diagnosis and long-term outcomes.

With respect to the approach to traumatic aneurysms, if the intent is to treat in an open surgical fashion, this is best accomplished at the time of initial cranial decompression. This is predicated on the high degree of suspicion of their presence. If this approach is not possible, every effort should be made to temporize the patient by endovascular means during the acute posttrauma period. Open surgery, if conducted in a delayed fashion, should be undertaken with the preoperative expectation that vessel sacrifice will be necessary. Coils, if present within the aneurysm, may aid in both the location of a distal aneurysm (intraoperative radiograph), and the manipulation of both the "dome" and the parent artery. That said, as in the setting of previously coiled spontaneous saccular aneurysms, tearing at the aneurysm neck is a distinct possibility, highlighting the need for good proximal and distal control. If coils are not present and the aneurysm is distal, the use of image guidance using fine-cut CT angiography should be considered.

Overall, because of the unpredictable nature of both vasospasm and traumatic aneurysms, there must be a willingness by the neurosurgeons involved with the care of these patients to treat safely and early.

\section{Disclosure}

The Comprehensive Neurosciences Program provided funding for the very early work analyzing the effect of traumatic vasospasm in this population (2004-2005).

Author contributions to the study and manuscript preparation include the following. Conception and design: RS Bell, R Armonda. Acquisition of data: RS Bell, RD Ecker, B Crandall. Analysis and interpretation of data: RS Bell, R Armonda. Drafting the article: RS Bell, R Armonda. Critically revising the article: RS Bell, RD Ecker, M Severson, J Wanebo, R Armonda. Reviewed final version of the manuscript and approved it for submission: RS Bell, R Armonda. Administrative/technical/material support: RS Bell. Study supervision: RS Bell, R Armonda. Other: B Crandall (direct care of patients).

The views expressed in this manuscript do not represent the official policy or opinion of the US Navy, Army, Department of Defense, or Government.

The authors certify that the document represents valid work; that if they used information derived from another source, they obtained all necessary approvals to use it and made appropriate acknowledgments in the document; and that each author takes public responsibility for it.

\section{Acknowledgments}

The authors are thankful for the service of all neurosurgeons deployed during OIF and OEF.

\section{References}

1. Aarabi B: Management of traumatic aneurysms caused by high-velocity missile head wounds. Neurosurg Clin N Am 6:775-797, 1995

2. Aarabi B: Traumatic aneurysms of brain due to high velocity missile head wounds. Neurosurgery 22:1056-1063, 1988

3. Achram M, Rizk G, Haddad FS: Angiographic aspects of traumatic intracranial aneurysms following war injuries. $\mathbf{B r}$ J Radiol 53:1144-1149, 1980

4. Amirjamshidi A, Rahmat H, Abbassioun K: Traumatic aneurysms and arteriovenous fistulas of intracranial vessels associated with penetrating head injuries occurring during war: principles and pitfalls in diagnosis and management. A survey of 31 cases and review of the literature. J Neurosurg 84:769-780, 1996

5. Anonymous: Vascular complications of penetrating brain injury. J Trauma 51 (Suppl 2):S26-S28, 2001

6. Armonda RA, Bell RS, Vo AH, Ling G, DeGraba TJ, Crandall B, et al: Wartime traumatic cerebral vasospasm: recent review of combat casualties. Neurosurgery 59:1215-1225, 2006

7. Bakshi A, Mahapatra AK: Basilar artery vasospasm after severe head injury: a preliminary transcranial Doppler ultrasound study. Natl Med J India 11:220-221, 1998

8. Bank WO, Nelson PB, Drayer BP, Wilkins RH, Rosenbaum AE: Traumatic aneurysm of the basilar artery. AJR Am J Roentgenol 130:975-977, 1978

9. Bauman RA, Ling G, Tong L, Januszkiewicz A, Agoston D, Delanerolle N, et al: An introductory characterization of a combat-casualty-care relevant swine model of closed head injury resulting from exposure to explosive blast. J Neurotrauma 26:841-860, 2009

10. Bell RS, Vo AH, Roberts R, Wanebo J, Armonda RA: Wartime traumatic aneurysms: acute presentation, diagnosis, and multimodal treatment of 64 craniocervical arterial injuries. Neurosurgery 66:66-79, 2010

11. Bell RS, Vo AH, Neal CJ, Tigno J, Roberts R, Mossop C, et al: Military traumatic brain and spinal column injury: a 5-year study of the impact blast and other military grade weaponry on the central nervous system. J Trauma 66 (4 Suppl):S104S111, 2009

12. Cairns CJS, Finfer SR, Harrington TJ, Cook R: Papaverine angioplasty to treat cerebral vasospasm following traumatic subarachnoid haemorrhage. Anaesth Intensive Care 31:8791,2003

13. Danziger J, Bloch S, Podlas H: Trauma of the arterial supply to the brain visualised by angiography. S Afr Med J 50:11-14, 1976

14. Ecker RD, Hopkins LN: Natural history of unruptured intracranial aneurysms. Neurosurg Focus 17(5):E4, 2004

15. Fukuda T, Hasue M, Ito H: Does traumatic subarachnoid hemorrhage caused by diffuse brain injury cause delayed ischemic brain damage? Comparison with subarachnoid hemorrhage caused by ruptured intracranial aneurysms. Neurosurgery 43:1040-1049, 1998

16. Hachemi M, Jourdan C, Di Roio C, Turjman F, Ricci-Franchi A, Mottolese C, et al: Delayed rupture of traumatic aneurysm after civilian craniocerebral gunshot injury in children. Childs Nerv Syst 23:283-287, 2007

17. Haddad F, Haddad GF, Taha J: Traumatic intracranial aneurysms caused by missiles: their presentation and management. Neurosurgery 28:1-7, 1991

18. Horowitz MB, Kopitnik TA, Landreneau F, Ramnani DM, Rushing EJ, George E, et al: Multidisciplinary approach to traumatic intracranial aneurysms secondary to shotgun and handgun wounds. Surg Neurol 51:31-42, 1999

19. Kordestani RK, Martin NA, McBride DQ: Cerebral hemodynamic disturbances following penetrating craniocerebral in- 
jury and their influence on outcome. Neurosurg Clin N Am 6:657-667, 1995

20. Lee JH, Martin NA, Alsina G, McArthur DL, Zaucha K, Hovda DA, et al: Hemodynamically significant cerebral vasospasm and outcome after head injury: a prospective study. J Neurosurg 87:221-233, 1997

21. Ludwiczak RW, Fogel LM: Posttraumatic aneurysm of the cervical segment of the internal carotid artery. Neuroradiology 10:179, 1975

22. Martin NA, Doberstein C, Alexander M, Khanna R, Benalcazar H, Alsina G, et al: Posttraumatic cerebral arterial spasm. J Neurotrauma 12:897-901, 1995

23. Martin NA, Patwardhan RV, Alexander MJ, Africk CZ, Lee $\mathrm{JH}$, Shalmon E, et al: Characterization of cerebral hemodynamic phases following severe head trauma: hypoperfusion, hyperemia, and vasospasm. J Neurosurg 87:9-19, 1997

24. McDonald EJ, Winestock DP, Hoff JT: The value of repeat cerebral arteriography in the evaluation of trauma. AJR Am J Roentgenol 126:792-797, 1976

25. Mira JM, Costa FA, Horta BL, Fabião OM: Risk of rupture in unruptured anterior communicating artery aneurysms: metaanalysis of natural history studies. Surg Neurol 66 (Suppl 3): S12-S19, 2006

26. Molyneux A, Kerr R, Stratton I, Sandercock P, Clarke M, Shrimpton J, et al: International Subarachnoid Aneurysm Trial (ISAT) of neurosurgical clipping versus endovascular coiling in 2143 patients with ruptured intracranial aneurysms: a randomised trial. Lancet 360:1267-1274, 2002

27. Molyneux AJ, Kerr RS, Yu LM, Clarke M, Sneade M, Yarnold JA, et al: International subarachnoid aneurysm trial (ISAT) of neurosurgical clipping versus endovascular coiling in 2143 patients with ruptured intracranial aneurysms: a randomised comparison of effects on survival, dependency, seizures, rebleeding, subgroups, and aneurysm occlusion. Lancet 366: 809-817, 2005

28. Nakstad P, Nornes H, Hauge HN: Traumatic aneurysms of the pericallosal arteries. Neuroradiology 28:335-338, 1986

29. Oertel M, Boscardin WJ, Obrist WD, Glenn TC, McArthur DL, Gravori T, et al: Posttraumatic vasospasm: the epidemiol- ogy, severity, and time course of an underestimated phenomenon: a prospective study performed in 299 patients. J Neurosurg 103:812-824, 2005

30. Romner B, Bellner J, Kongstad P, Sjöholm H: Elevated transcranial Doppler flow velocities after severe head injury: cerebral vasospasm or hyperemia? J Neurosurg 85:90-97, 1996

31. Soustiel JF, Shik V, Feinsod M: Basilar vasospasm following spontaneous and traumatic subarachnoid haemorrhage: clinical implications. Acta Neurochir (Wien) 144:137-144, 2002

32. Taneda M, Kataoka K, Akai F, Asai T, Sakata I: Traumatic subarachnoid hemorrhage as a predictable indicator of delayed ischemic symptoms. J Neurosurg 84:762-768, 1996

33. Vajramani GV, Chandramouli BA, Jayakumar PN, Kolluri S: Evaluation of posttraumatic vasospasm, hyperaemia, and autoregulation by transcranial colour-coded duplex sonography. Br J Neurosurg 13:468-473, 1999

34. Vardiman AB, Kopitnik TA, Purdy PD, Batjer HH, Samson DS: Treatment of traumatic arterial vasospasm with intraarterial papaverine infusion. AJNR Am J Neuroradiol 16:319321,1995

35. White PM, Wardlaw JM: Unruptured intracranial aneurysms. J Neuroradiol 30:336-350, 2003

36. Zubkov AY, Lewis AI, Raila FA, Zhang J, Parent AD: Risk factors for the development of post-traumatic cerebral vasospasm. Surg Neurol 53:126-130, 2000

37. Zubkov AY, Pilkington AS, Bernanke DH, Parent AD, Zhang J: Posttraumatic cerebral vasospasm: clinical and morphological presentations. J Neurotrauma 16:763-770, 1999

Manuscript submitted January 15, 2010.

Accepted February 16, 2010.

Address correspondence to: Randy S. Bell, M.D., Cerebrovascular Neurosurgery and Interventional Neuroradiology, NAVMED MPT\&E, and Department of Neurosurgery, National Naval Medical Center, 8901 Wisconsin Avenue, Bethesda, Maryland 20889. email: randy.s.bell@us.army.mil. 